\title{
Collimation of sound assisted by acoustic surface waves
}

\author{
J. CHRISTENSEN ${ }^{1}$, A. I. FERNANDEZ-DOMINGUEZ¹ , F. DE LEON-PEREZ², L. MARTIN-MORENO² \\ AND F. J. GARCIA-VIDAL ${ }^{1 *}$ \\ ${ }^{1}$ Departamento de Fisica Teorica de la Materia Condensada, Universidad Autonoma de Madrid, Madrid 28049, Spain \\ ${ }^{2}$ Departamento de Fisica de la Materia Condensada-ICMA, Universidad de Zaragoza, Zaragoza 50009, Spain \\ *e-mail: fj.garcia@uam.es
}

The discovery of the phenomenon of extraordinary optical transmission through a two-dimensional array of subwavelength holes in a metallic film ${ }^{1}$ has opened a new line of research within optics. The key role played by surface plasmons in transferring light efficiently from the input side of the metal film to the output region was soon realized. This fundamental knowledge enabled extension of this surface-plasmon ability to achieve extraordinary optical transmission and strong collimation of light in a single hole surrounded by a finite periodic array of indentations ${ }^{2}$. Here, we show how these ideas developed for electromagnetic radiation can be transferred to other classical waves such as acoustic waves.

In analogy with the electromagnetic case, the main actors necessary for the appearance of both enhanced transmission and collimation in acoustics are the acoustic surface waves (ASWs). There are many different types of ASW (see, for example ref. 3), depending on the nature of the two media forming the interface (solid-solid, solid-fluid, fluid-fluid and so on). ASWs have been thoroughly studied in the past owing to both their fundamental interest and technological applications. In particular, ASWs such as Rayleigh-type waves appearing at the interface between a solid material and a fluid are now widely used in the so-called SAW devices. Here, we focus on ASWs appearing at a fluidfluid interface, although our findings could easily be translated to other elastic surface waves. The condition for the existence of an ASW propagating at the interface between two semi-infinite fluids is very similar to the equation governing the presence of a surface electromagnetic mode running at the interface between two dielectric media:

$$
\frac{k_{z}^{\mathrm{I}}}{\rho^{\mathrm{I}}}+\frac{k_{z}^{\mathrm{II}}}{\rho^{\mathrm{II}}}=0,
$$

where $k_{z}^{\mathrm{I}}$ and $k_{z}^{\mathrm{II}}$ are the inverse of the decay lengths of the ASW in media I and II, respectively, and $\rho^{\mathrm{I}}$ and $\rho^{\mathrm{II}}$ are the corresponding mass densities. Note that the mass density plays the same role as the dielectric constant in the electromagnetic case. It is clear that if the two media have positive mass densities, there is no ASW running at the I-II interface. One possibility for generating ASWs is to place a fluid layer of uniform thickness on top of a semiinfinite fluid. Here, we consider a similar alternative, in which ASWs are created by periodically corrugating the interface between a rigid body and a fluid. The simplest way of doing this is by perforating a one-dimensional (1D) array of grooves, as seen in

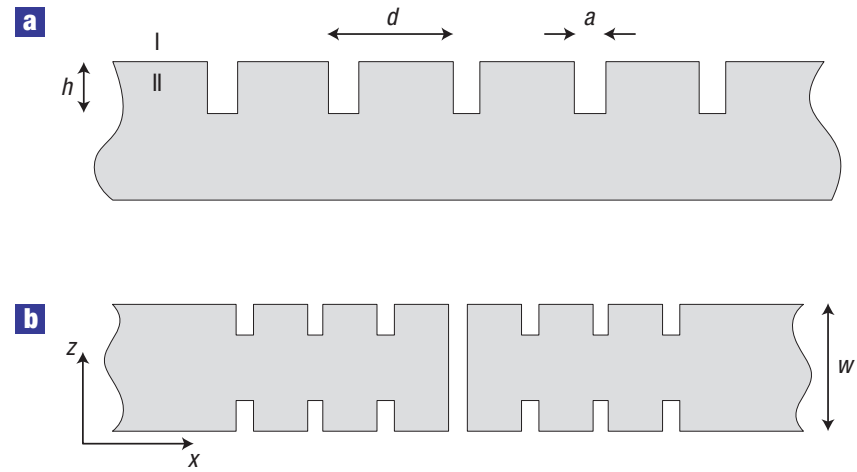

Figure 1 Geometry-induced ASWs and structure under study. a, Schematic diagram of a 1D periodic array (period $d$ ) of grooves (side a and height $h$ ) drilled in the surface of a perfect rigid body surrounded by air. $\mathbf{b}, \mathrm{A} 1 \mathrm{D}$ slit of side a perforated on a perfect rigid body of thickness $w$ flanked to the left and right by finite arrays of grooves. Definitions of axes $x$ and $z$ used in the calculations are also shown. The structure is translationally invariant in the $y$ direction.

Fig. 1a. The fluid in region II of thickness $h$ can be characterized effectively by a mass density that is different to the one associated with the fluid in semi-infinite medium I. In other words, we could say that medium II is an acoustic metamaterial ${ }^{4}$. To the best of our knowledge, the idea of corrugating an interface to create a fluid-fluid ASW was first proposed ${ }^{5}$ and verified experimentally ${ }^{6}$ by Kelders and co-workers in connection with ultrasonic surface waves in porous media. Similar ideas for ASWs in solid media were previously suggested by Gulyaev ${ }^{7}$.

In the case of a corrugated perfect rigid body, the dispersion relation (relation between frequency, $\omega$, and momentum, $k$ ) of these geometry-induced ASWs is controlled by the geometrical parameters of the array of grooves and the density of the fluid. When the width of the grooves, $a$, is much smaller than the period of the array, $d$, a good approximation for $k(\omega)$ is given by:

$$
k=k_{0} \sqrt{1+\frac{a^{2}}{d^{2}} \tan ^{2} k_{0} h},
$$

where $k_{0}=\omega / c_{\mathrm{S}}$, with $c_{\mathrm{S}}$ being the velocity of sound in medium I. The periodic corrugation creates an ASW but, at the same time, can 


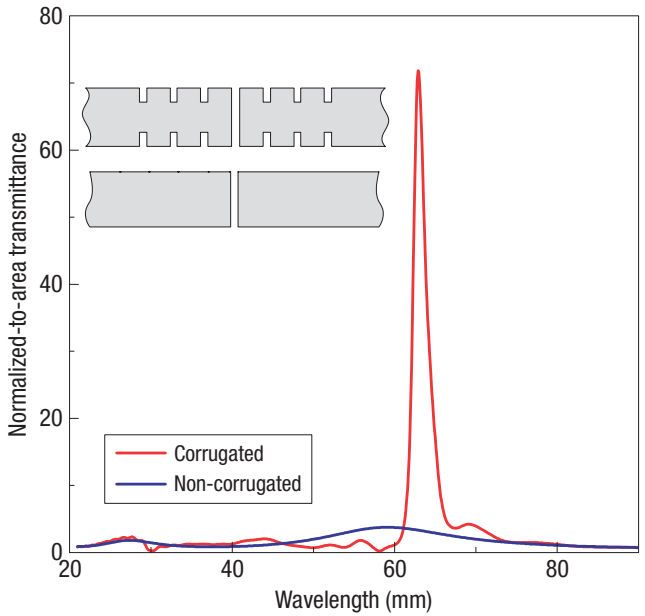

Figure 2 Resonant transmission of sound through a single subwavelength aperture. Normalized-to-area transmittance (red curve) versus wavelength for a normal incident acoustic plane wave propagating in air and impinging at a single slit $(a=5 \mathrm{~mm})$ drilled in a solid slab. The aperture is flanked by 10 grooves ( $d=60 \mathrm{~mm}, h=9 \mathrm{~mm}$ ) on a solid of thickness $w=22 \mathrm{~mm}$. The transmission spectrum for a single slit is also shown (blue curve).

provide the necessary parallel momentum to allow the coupling of an incident acoustic plane wave with the ASW and vice versa.

Resonant transmission of sound in periodic acoustic structures aided by the excitation of localized modes has been analysed before ${ }^{8,9}$. Here, we use the properties of the geometry-induced ASWs to enhance the transmission through a single subwavelength aperture and to collimate sound waves. As shown below, these two phenomena can be achieved by placing finite arrays of indentations around the aperture (see Fig. 1b). To calculate the transmission properties of this structure, we have applied a numerical method on the basis of a modal expansion of the pressure field. By applying the matching conditions (continuity of the pressure only at the openings and continuity of the velocity everywhere), it is possible to extract the pressure and velocity fields in all space. Figure 2 shows the calculated normalized-to-area transmittance versus wavelength for an incident acoustic plane wave through a $1 \mathrm{D}$ slit flanked by periodic arrays of 10 grooves to both its left and right. A resonant peak appearing at a wavelength close to the period of the array clearly dominates the spectrum. For sound of that particular wavelength, the transmitted intensity is 70 times larger than the one impinging directly at the slit opening. This means that a significant portion $(30 \%)$ of the intensity incident on the finite array of grooves is collected by the ASW and funnelled through the central slit.

Accompanying the resonant transmission, the sound wave at resonance emerges from the structure exciting an ASW (the fingerprints of this running surface wave are clearly seen in Fig. 3b). This ASW is then scattered by the grooves and the interference between these scattered waves and the sound wave that was just diffracted by the slit results in the emergence of the strongly collimated beam in the far-field region (see Fig. 3a). Note that in contrast to previous studies achieving lateral compression or collimation of ASWs, we use surface waves to collimate an acoustic plane wave that impinges perpendicular to the structure.

The fact that both enhanced transmission and collimation phenomena occur also in acoustics opens up many exciting possibilities in the design of new acoustic devices. We foresee that our finding will lead to applications for mechanical

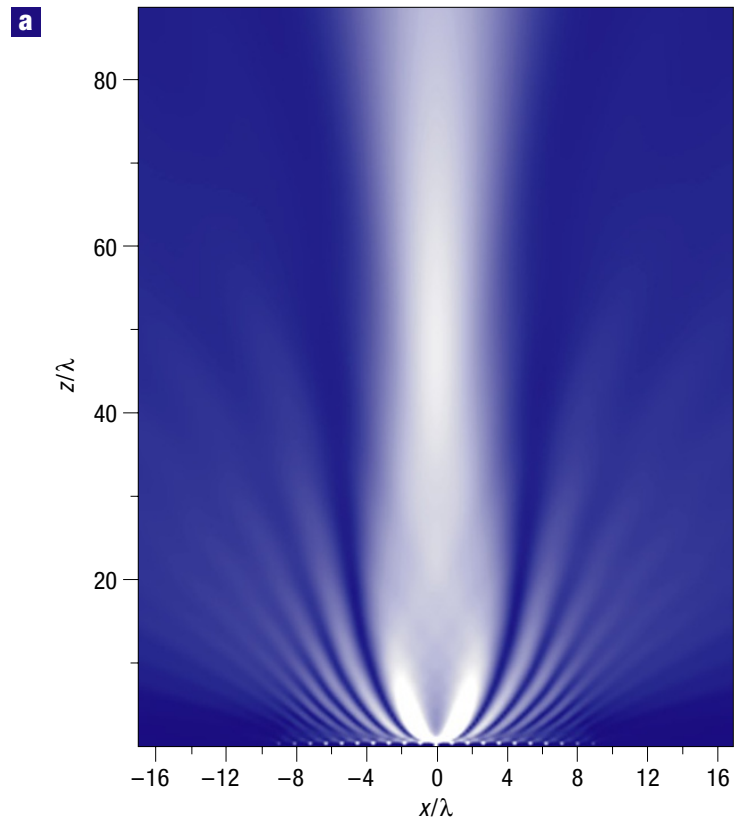

b
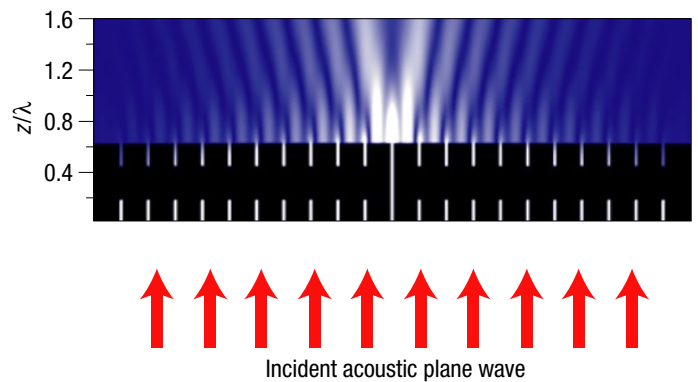

Figure 3 Collimation of sound assisted by ASWs. a, Pressure-field-amplitude spatial pattern in the $x z$ plane evaluated at resonance. The collimated beam has an elongated focus in the far-field region. $\mathbf{b}$, Magnified view of panel a showing the region near the output side of the slit, highlighting the excitation of an ASW.

filtering, in non-destructive testing of materials ${ }^{10}$ and in medical ultrasound instrumentation ${ }^{11}$.

Received 28 June 2007; accepted 28 September 2007; published 28 October 2007.

\section{References}

1. Ebbesen, T. W., Lezec, H. J., Ghaemi, H. F., Thio, T. \& Wolff, P. A. Extraordinary optical transmission through subwavelength hole arrays. Nature 391, 667-669 (1998).

2. Lezec, H. J. et al. Beaming light from a subwavelength aperture. Science 297, 820-822 (2002).

3. Hess, P. Surface acoustic waves in materials science. Phys. Today 42-47 (March 2002).

4. Fang, N. et al. Ultrasonic metamaterials with negative modulus. Nature Mater. 5, 452-456 (2006).

5. Kelders, L., Allard, J. F. \& Lauriks, W. Ultrasonic surface waves above rectangular-groove gratings. J. Acoust. Soc. Am. 103, 2730-2733 (1998).

6. Kelders, L., Lauriks, W. \& Allard, J. F. Surface waves above thin porous layers saturated by air at ultrasonic frequencies. J. Acoust. Soc. Am. 104, 882-889 (1998).

7. Gulyaev, Y. V. \& Plesskii, V. P. Slow acoustic surface waves in solids. Sov. Tech. Phys. Lett. 3 , 87-88 (1977).

8. Zhang, $X$. Acoustic resonant transmission through acoustic gratings with very narrow slits: multiple-scattering numerical simulations. Phys. Rev. B 71, 241102(R) (2005).

9. Sainidou, R. \& Stefanou, N. Guided and quasiguided elastic waves in phononic crystal slabs. Phys. Rev. B 73, 184301 (2006).

10. Fink, M. Ultrasound puts materials to the test. Phys. World 41-45 (February 1998).

11. Kennedy, J. E., Ter Haar, G. R. \& Cranston, D. High intensity focused ultrasound: Surgery of the future? Br. J. Radiol. 76, 590-599 (2003).

\section{Acknowledgements}

J.C. acknowledges the encouragement of H. Kamath. Financial support from the Spanish MEC under contract MAT2005-06608-C02 is gratefully acknowledged.

Correspondence and requests for materials should be addressed to F.J.G.-V.

Reprints and permission information is available online at http://npg.nature.com/reprintsandpermissions/ 